\title{
Uniqueness of differential characters and differential K-theory via homological algebra
}

\author{
Ishan Mata
}

\begin{abstract}
In Proc Math Sci 129, 70(219), Rakesh Pawar considers and solves a certain diagram extension problem. In this note, we observe that the existence and uniqueness of differential characters (defined as objects which fit into a certain hexagon diagram) follow directly from Rakesh Pawar's results. This provides an alternate proof of a weaker version of J. Simons and D. Sullivan's results (Journal of Topology, 2008, 1:45-56). Further, this approach directly shows that the hexagon diagram uniquely determines the differential K-theory groups upto an isomorphism.
\end{abstract}

\section{Introduction}

In the seminal work [1], J. Cheeger and J. Simons introduced and developed the theory of differential characters. For a fixed smooth manifold $M$, they defined the abelian group of differential characters $\hat{H}^{k}(M ; \mathbb{R} / \mathbb{Z})$ as 1

$$
\hat{H}^{k}(M ; \mathbb{R} / \mathbb{Z})=\left\{f: \operatorname{Hom}\left(Z_{k-1}(M), \mathbb{R} / \mathbb{Z}\right) \mid f \circ \delta \in \Omega^{k}\right\} .
$$

Cheeger and Simons show that there are short exact sequences:

$$
0 \rightarrow H^{k-1}(M ; \mathbb{R} / \mathbb{Z}) \stackrel{j}{\rightarrow} \hat{H}^{k}(M ; \mathbb{R} / \mathbb{Z}) \stackrel{\text { curv }}{\longrightarrow} \Omega_{0}^{k}(M) \rightarrow 0,
$$

and

$$
0 \rightarrow \frac{\Omega^{k-1}(M)}{\Omega_{0}^{k-1}(M)} \stackrel{i}{\rightarrow} \hat{H}^{k}(M ; \mathbb{R} / \mathbb{Z}) \stackrel{c}{\rightarrow} H^{k}(M ; \mathbb{Z}) \rightarrow 0 .
$$

Here $\Omega_{0}^{k}(M)$ stands for closed degree $k$ forms with integral periods. They further showed that the composition $H^{k-1}(M ; \mathbb{R} / \mathbb{Z}) \stackrel{j}{\rightarrow} \hat{H}^{k}(M ; \mathbb{R} / \mathbb{Z}) \stackrel{c}{\rightarrow}$

\footnotetext{
${ }^{1}$ Our convention of degrees differs from the one in original paper [1] where this group is called $\hat{H}^{k-1}(M ; \mathbb{R} / \mathbb{Z})$.
} 
$H^{k}(M ; \mathbb{Z})$ is $-B$ where $B$ is the Bockstein map; and the composition $\frac{\Omega^{k-1}(M)}{\Omega_{0}^{k-1}(M)} \stackrel{i}{\rightarrow}$ $\hat{H}^{k}(M ; \mathbb{R} / \mathbb{Z}) \stackrel{\text { curv }}{\longrightarrow} \Omega_{0}^{k}(M)$ is the exterior derivative $d$.

There are various other constructions of differential refinements of ordinary singular cohomology in the literature, see for example [2, 3, 4, 5, 6]. It is natural to ask whether these constructions are equivalent. In [7], Simons and Sullivan provide an axiomatic characterization of differential cohomology. They show that the following hexagon diagram with exact diagonals [7] :

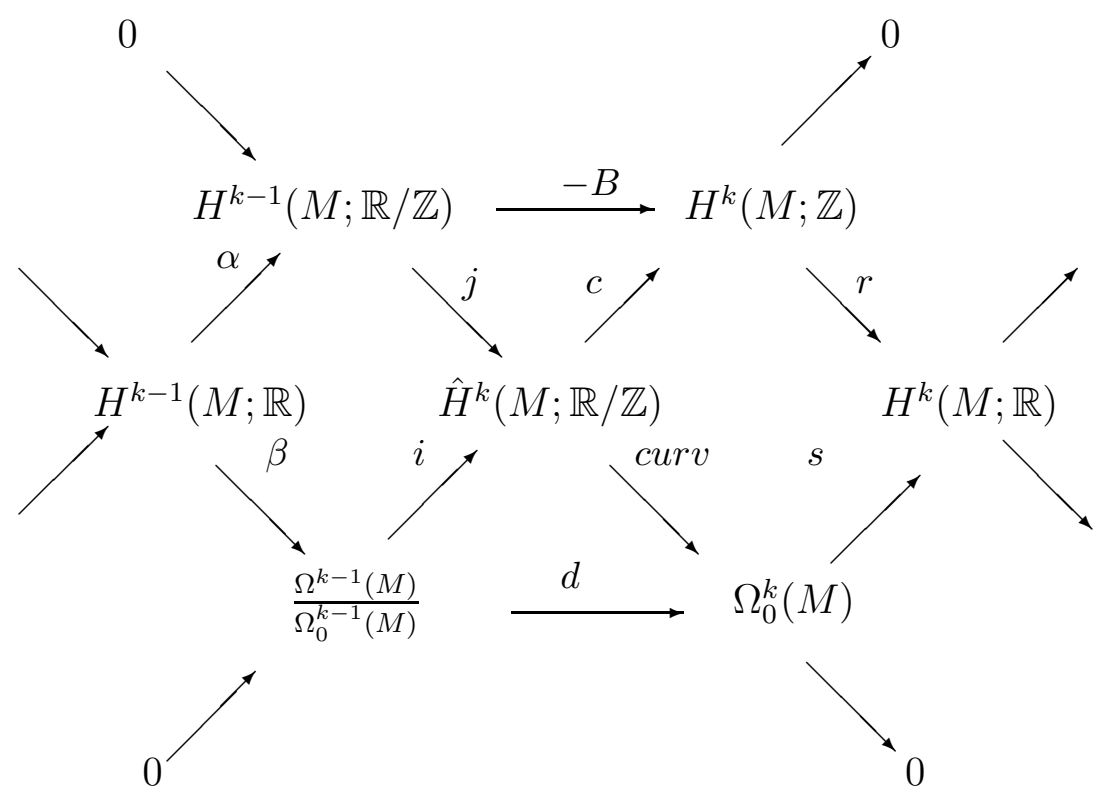

Differential cohomology hexagon diagram

uniquely characterizes the ordinary differential cohomology functor upto a natural equivalence (for a precise statement, see proposition 6). The long exact sequence of the upper arrows is the long exact sequence in cohomology corresponding to the short exact sequence $0 \rightarrow \mathbb{Z} \rightarrow \mathbb{R} \rightarrow \mathbb{R} / \mathbb{Z} \rightarrow 0$. The map $\beta$ and $s$ are induced by the the de-Rham morphism.

This axiomatization of differential cohomology is useful since it establishes that different constructions of differential refinements of ordinary singular cohomology are essentially equivalent. For example, since the Deligne cohomology functor [3] - defined as hypercohomology of a certain complex- fits in the hexagon diagram [2], it follows that the Cheeger-Simons differential character functor is naturally equivalent to the Deligne cohomology functor via an equivalence compatible with the diagonal morphisms in the respective hexagon diagrams.

Just as ordinary differential cohomology admits a differential refinement, so do other generalised cohomology theories. Given a generalised cohomology 
theory represented by a spectrum, Hopkins and Singer gave a prescription [8] for constructing its differential refinement. In particular one can construct a differential version of K-theory using their prescription. In [9], Simons and Sullivan construct another model of differential K-theory for compact manifolds in terms of structured vector bundles. They show that their model of differential K-theory fits in the following hexagon diagram with exact diagonals :

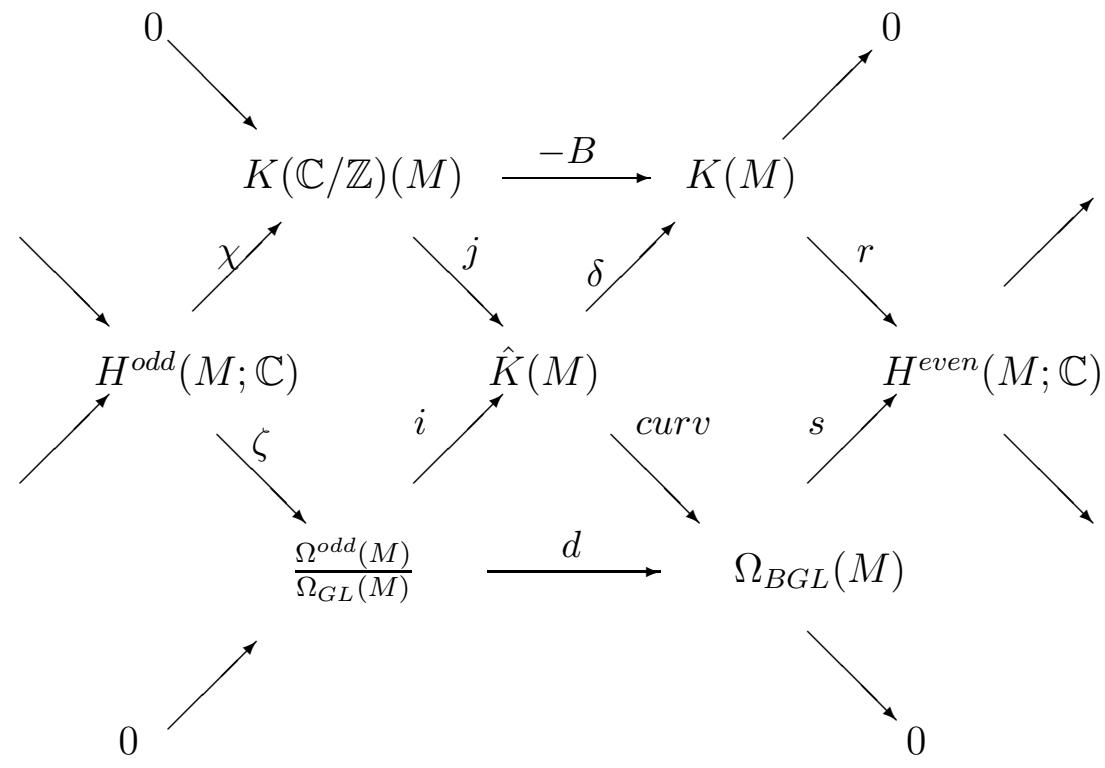

Differential K-theory hexagon diagram

They ask whether, like in the case of differential characters,

Question 1. Does the above hexagon diagram determine the differential Ktheory functor (from the category of compact manifolds to the category of abelian groups) upto a natural equivalence compatible with the respective diagonal morphisms ?

In [10, Rakesh Pawar finds necessary and sufficient conditions for the diagram 


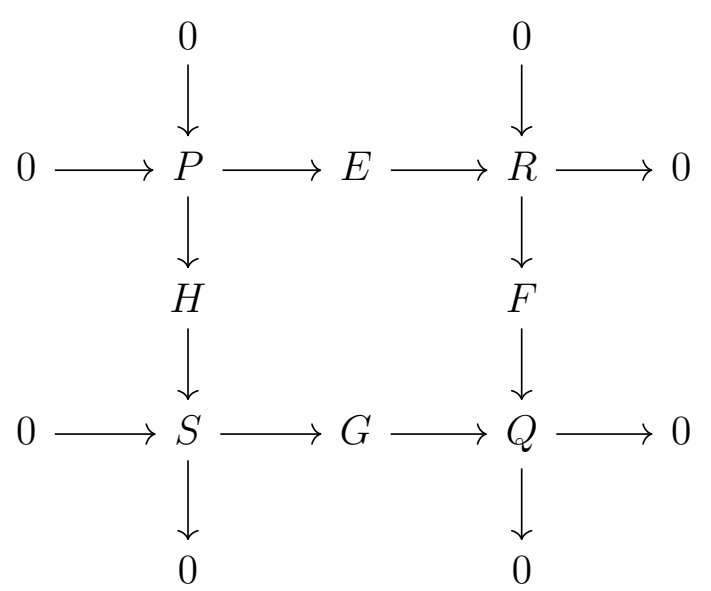

Diagram 1

with short exact rows and columns to extend to

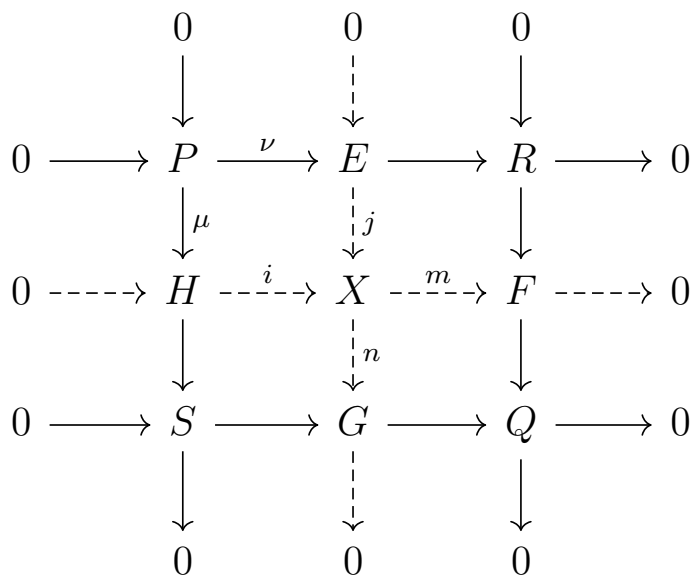

Diagram 2

with short exact rows and columns. He further gives conditions for uniqueness of such extensions.

In this modest note, we wish to highlight that the results of Rakesh Pawar directly imply (see proposition 7) the existence and uniqueness of differential character groups $\hat{H}^{k}(M)$ (defined as objects which fit into the hexagon diagram with exact diagonals). Uniqueness of the functor $\hat{H}^{k}(-; \mathbb{R} / \mathbb{Z})$ is a stronger result, for which we do not have a complete proof. However, we state a condition 8 which implies the full Simons-Sullivan result.

Similarly we note in proposition 10 that for any compact manifold $M$, the differential K-theory groups are uniquely determined upto an isomorphism compatible with the respective diagonal maps, thereby partially answering a 
question 1 of Simons and Sullivan. We give necessary and sufficient conditions 11 for an affirmative answer to the Simons-Sullivan question in full generality.

This note is organised as follows. In section 2 we summarize the theorems of the article [10] that we need for our purposes. In 3 we state the uniqueness results for differential characters and differential K-theory. These uniqueness results are a direct corollary of Rakesh Pawar's purely homological algebraic results involving no topology or geometry. Interestingly, for this reason, this approach may potentially admit an adaptation for axiomatising other generalised differential cohomology theories.

Axiomatic characterization of generalised differential cohomology theories with fiber integration, and their uniqueness has been discussed in [1].

\section{Statement of Rakesh Pawar's results}

In this section, we summarize the results of [10] that we need for present purposes. Let us begin by recalling some standard preliminary definitions and results from homological algebra (see, for example, [12, 13]). If $\mathcal{A}$ is an abelian category with enough projectives, then for any two objects $P, Q$ in $\mathcal{A}$, one can consider the groups $\operatorname{Ext}^{n}(Q, P)$ as the derived functor of the Hom functor.

Alternatively, one can consider the group of Yoneda extensions of $P$ by $Q$ as follows. Consider the set of long exact sequences $\zeta: 0 \rightarrow P \rightarrow X_{n} \rightarrow$ $\cdots \rightarrow X_{1} \rightarrow Q \rightarrow 0$. If $\zeta^{\prime}: 0 \rightarrow P \rightarrow X_{n}^{\prime} \rightarrow \cdots \rightarrow X_{1}^{\prime} \rightarrow Q \rightarrow 0$ is another such extension, a map $f: \zeta \rightarrow \zeta^{\prime}$ is a collection of maps $f_{i}: X_{i} \rightarrow X_{i}^{\prime}$ such that the diagram

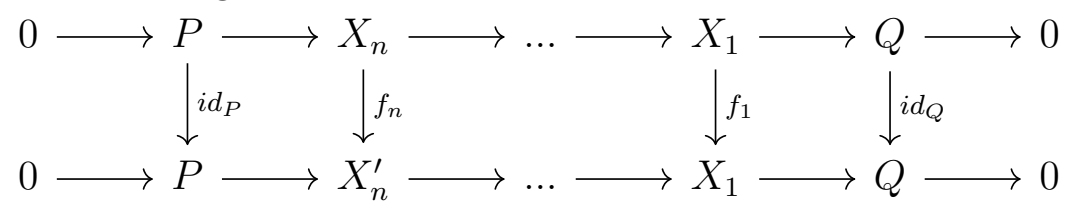

commutes. Define an equivalence relation $\zeta \sim \eta \Longleftrightarrow \exists$ a finite zigzag chain $\zeta \rightarrow \alpha_{1} \leftarrow \alpha_{2} \rightarrow \alpha_{3} \leftarrow \cdots \rightarrow \eta$. Quotient of the set of extensions considered above by this equivalence relation gives us the set of Yoneda extensions $\operatorname{Ext}_{\text {Yoneda }}^{n}(Q, P)$. On this set, define addition as $\zeta+\zeta^{\prime}=[0 \rightarrow$ $\left.P \rightarrow Y_{n} \rightarrow X_{n-1}^{\prime} \oplus X_{n-1} \rightarrow \cdots \rightarrow X_{2}^{\prime} \oplus X_{2} \rightarrow Y_{1} \rightarrow Q \rightarrow 0\right]$. Here $Y_{1}$ is the pullback $X_{1} \times{ }_{Q} X_{1}^{\prime}$, and $Y_{n}$ is the quotient by a skew diagonal copy of $P$, of the pushout of $P \rightarrow X_{n}$ and $P \rightarrow X_{n}^{\prime}$. The set $\operatorname{Ext}_{Y \text { oneda }}^{n}(Q, P)$ becomes an abelian group under this operation. If the category $\mathcal{A}$ has enough projectives, then $\operatorname{Ext}_{\text {Yoneda }}^{n}(Q, P)$ is isomorphic to $\operatorname{Ext}^{n}(Q, P)$ (see, for example, section 3.4 of [12]). Throughout this article we shall assume that the category $\mathcal{A}$ has enough projectives. 
Proposition 1. (Rakesh Pawar) : Let $\mathcal{A}$ be an abelian category. Let Diagram 1 have exact rows and columns of objects of $\mathcal{A}$. The diagram 1 extends to diagram 2 with exact rows and columns if and only if the Baer sum of $[E] \cup[F]$ and $[H] \cup[G]$ is zero in $\operatorname{Ext}^{2}(Q, P)$.

In [10], this proposition is stated for small categories, however as noted in the remark 3.3 of [10], the result holds good for general abelian categories. Stating the uniqueness theorem requires a bit of background. First pull back the exact sequence $0 \rightarrow R \rightarrow F \rightarrow Q \rightarrow 0$ by the map $G \rightarrow Q$ to get :

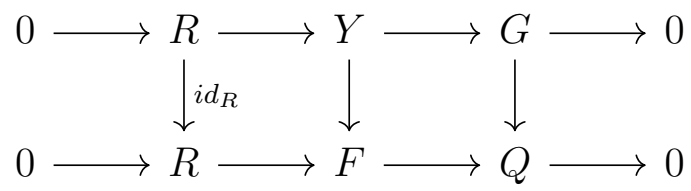

Applying the Snake lemma, Rakesh Pawar obtains

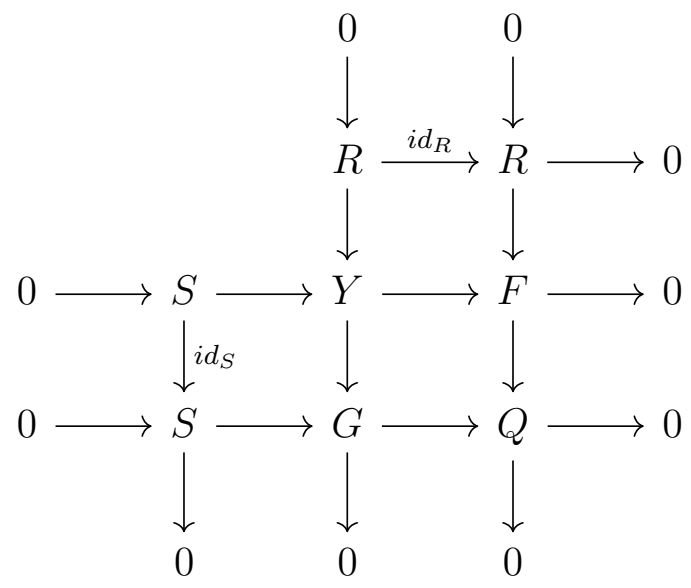

- The injective maps $R \rightarrow Y$, and $S \rightarrow Y$ induce a map $R \oplus S$ induce a short exact sequence $0 \rightarrow R \oplus S \rightarrow Y \rightarrow Q \rightarrow 0$. Applying the functor $\operatorname{Hom}(-, P)$, one obtains the long exact sequence

$$
\cdots \rightarrow \operatorname{Hom}(R \oplus S, P) \stackrel{\alpha}{\rightarrow} \operatorname{Ext}^{1}(Q, P) \stackrel{\beta}{\rightarrow} \operatorname{Ext}^{1}(Y, P) \rightarrow \cdots
$$

Proposition 2. (Rakesh Pawar) If the map $\alpha$ is surjective, then $[X] \in$ $\operatorname{Ext}^{1}(Y, P)$ is unique.

Thus if $X_{1}$ is another abelian group together with maps $i_{1}, j_{1}, m_{1}, n_{1}$, then $[X]=\left[X_{1}\right] \in \operatorname{Ext}^{1}(Y, P)$. Equivalently there is an abelian group isomorphism $\phi: X \rightarrow X_{1}$ such that the diagram 


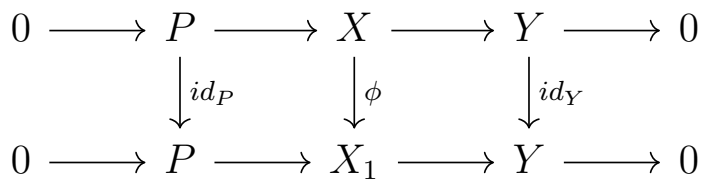

commutes. Since $Y$ is the pullback

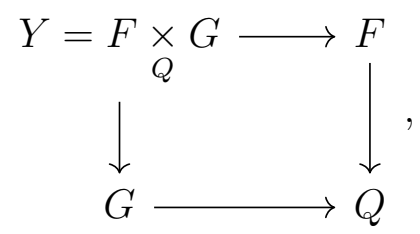

it follows that the morphism $\phi$ is compatible with $\left(m, m_{1}\right)$ and $\left(n, n_{1}\right)$ i.e. $m_{1} \circ \phi=m$ and $n_{1} \circ \phi=n$, and that $\left.\phi \circ i\right|_{P}=\left.i_{1}\right|_{P},\left.\phi \circ j\right|_{P}=\left.j_{1}\right|_{P}$. (Here we are considering $P$ as a subgroup of $H$ via $\mu$, and of $E$ via $\nu$.) Alternatively we could say that $\phi \circ i \circ \mu=i_{1} \circ \mu$, and $\phi \circ j \circ \nu=j_{1} \circ \nu$. However, we need a stronger compatibility result for our purposes : $\phi \circ i=i_{1}$, and $\phi \circ j=j_{1}$. We obtain this in the next section.

\section{Existence and uniqueness results for differ- ential cohomology theories}

Let $\left(X_{1}, i_{1}, j_{1}, m_{1}, n_{1}\right)$ and $\left(X_{2}, i_{2}, j_{2}, m_{2}, n_{2}\right)$ be two extensions of Diagram 1. Let us say that an isomorphism $\phi: X_{1} \rightarrow X_{2}$ is a compatible isomorphism between these two extensions if $\phi \circ i_{1}=i_{2}, \phi \circ j_{1}=j_{2}, m_{2} \circ \phi=m_{1}$, and $n_{2} \circ \phi=$ $n_{1}$. The following proposition gives a necessary and sufficient criterion for an extension (assuming it exists) to be determined upto such equivalence.

Proposition 3. Suppose $\left(X_{1}, i_{1}, j_{1}, m_{1}, n_{1}\right)$ is an extension of Diagram 1. Let $E_{1}=j_{1}(E) \subset X_{1}$, and $H_{1}=i_{1}(H) \subset X_{1}$. The the following are equivalent :

1. For any other extension $\left(X_{2}, i_{2}, j_{2}, m_{2}, n_{2}\right)$ of the diagram, there exists an isomorphism $\phi: X_{1} \rightarrow X_{2}$ compatible with the two extensions.

2. The map $\operatorname{Hom}(R \oplus S, P) \stackrel{\alpha}{\rightarrow} \operatorname{Ext}^{1}(Q, P)$ is surjective, and every homomorphism $\lambda: E_{1}+H_{1} \rightarrow P$ which vanishes on $P_{1} \subset E_{1}+H_{1}$ admits an extension $\Lambda: X_{1} \rightarrow P$.

Proof. We shall show that $(2) \Longrightarrow(1)$. The other direction follows by retracing the steps of the proof.

By Rakesh Pawar's result 2, there is an isomorphism $\phi: X_{1} \rightarrow X_{2}$ such 
that $m_{2} \circ \phi=m_{1}$, and $n_{2} \circ \phi=n_{1}$. The strategy is to find a morphism $\eta: X_{1} \rightarrow X_{2}$ such that the morphism $\phi^{\prime} \equiv \phi+\eta$ is a compatible isomorphism i.e. $\phi^{\prime} \circ i_{1}=i_{2}, \phi^{\prime} \circ j_{1}=j_{2}, m_{2} \circ \phi^{\prime}=m_{1}$, and $n_{2} \circ \phi^{\prime}=n_{1}$.

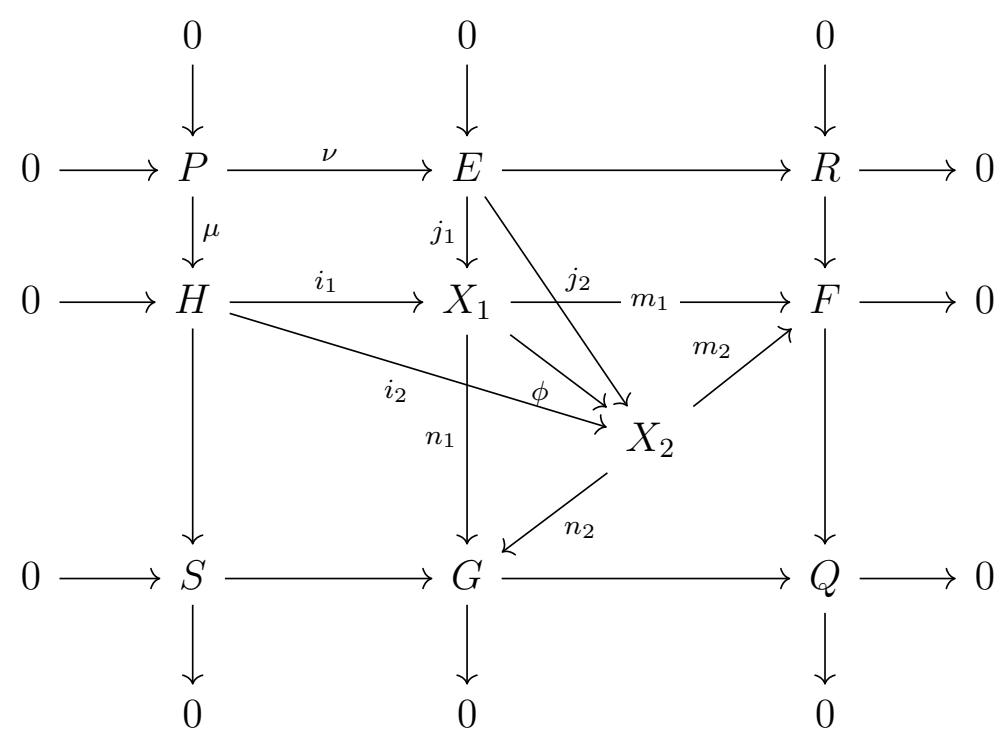

For convenience, let us denote $P_{1} \equiv i_{1} \circ \mu(P)=j_{1} \circ \nu(P) \subset X_{1}$, and $P_{2} \equiv$ $i_{2} \circ \mu(P)=j_{2} \circ \nu(P) \subset X_{2}$. Similarly let $E_{1}=j_{1}(E), E_{2}=j_{2}(E), H_{1}=i_{1}(H)$, and $H_{2}=i_{2}\left(H_{\tilde{j}}\right)$.

Now consider $\tilde{j}=j_{2}-\phi \circ j_{1}: E \rightarrow X_{2}$. Note that $m_{2} \circ \tilde{j}=m_{2} \circ j_{2}-m_{2} \circ \phi \circ j_{1}=$ $m_{2} \circ j_{2}-m_{1} \circ j_{1}=0$, and $n_{2} \circ \tilde{j}=n_{2} \circ j_{2}-n_{2} \circ \phi \circ j_{1}=m_{2} \circ j_{2}-n_{1} \circ j_{1}=0$. Thus $\tilde{j}(E) \subset P_{2}$. Similarly $\tilde{i}(H) \subset P_{2}$. Also note that since $\left.\phi \circ i_{1}\right|_{P}=\left.i_{2}\right|_{P}$ and $\left.\phi \circ j_{1}\right|_{P}=\left.j_{2}\right|_{P}$ (by the discussion after proposition 2), we conclude that $\left.\tilde{j}\right|_{P}=0=\left.\tilde{i}\right|_{P}$. Hence, $\tilde{i}+\tilde{j}: E_{1}+H_{1} \rightarrow X_{2}$ is a well defined abelian group homomorphism taking values in $P_{2}$. Here we have identified $E$ with $E_{1}$, and $H$ with $H_{1}$, for notational simplicity we use the same notation for the maps $\tilde{i}$ and $\tilde{j}$. We thus have a commutative diagram :

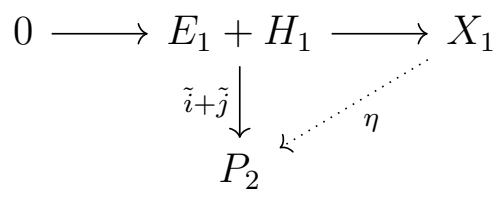

By hypothesis, there exists an extension $\eta: X_{1} \rightarrow X_{2}$. Let $\phi^{\prime} \equiv \phi+\eta$. Then $\phi^{\prime} \circ i_{1}=\phi \circ i_{1}+\eta \circ i_{1}=\phi \circ i_{1}+\left(i_{2}-\phi \circ i_{1}\right)=i_{2}$, and $\phi^{\prime} \circ j_{1}=\phi \circ j_{1}+\eta \circ j_{1}=$ $\phi \circ j_{1}+\left(j_{2}-\phi \circ j_{1}\right)=j_{2}$. Further since $\eta$ takes values in $P_{2}, m_{2} \circ \eta=0$. Thus $m_{2} \circ \phi^{\prime}=m_{2} \circ \phi+m_{2} \circ \eta=m_{2} \circ \phi=m_{1}$, and similarly $n_{2} \circ \phi=n_{1} . \phi^{\prime}$ is an isomorphism by the three lemma. Hence $\phi^{\prime}$ is a required isomorphism. 
Corollary 4. If $P$ is an injective object, there exists an extension of the diagram 1. Further for any two such extensions, there exists a compatible isomorphism between them.

Let Man be the category of smooth manifolds and smooth maps between them, and let $\mathbf{A b}$ be the category of abelian groups and group homomorphisms.

\subsection{The case of differential characters}

As noted in the introduction, the Deligne cohomology groups defined as hypercohomology of a certain double complex [3] are isomorphic to the differential character groups $\hat{H}^{k}(M ; \mathbb{R} / \mathbb{Z})$ defined by Cheeger-Simons. Similarily, the de Rham-Federer currents [4, 5] too provide a model of differential cohomology. In order to compare various models, it is important to axiomatically characterize ordinary differential cohomology. In [7] Simons and Sullivan define :

Definition 5. A functor $\hat{H}^{k}$ from $\boldsymbol{M a n}{ }^{o p}$ to $\boldsymbol{A b}$, together with natural transformations $i, j, c$, curv is called a differential character functor if the following diagram in Fun $\left(\boldsymbol{M a n}^{o p}, \boldsymbol{A b}\right)$

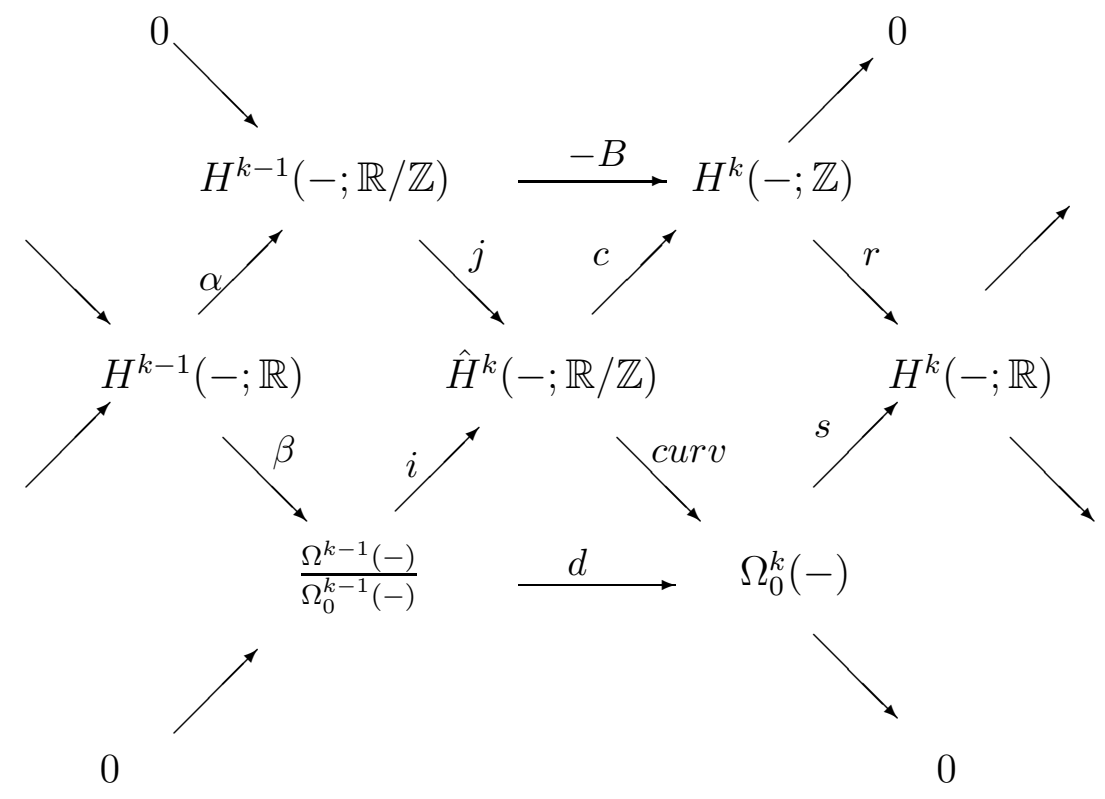

commutes and has exact diagonals.

and prove 
Proposition 6. If $\hat{H}_{1}(-)$ and $\hat{H}_{2}(-)$ are two differential character functors from $\boldsymbol{M a n}{ }^{o p}$ to $\boldsymbol{A} \boldsymbol{b}$ together with the natural transformations $\left(i_{1}, j_{1}, c_{1}\right.$, curv $\left._{1}\right)$ and $\left(i_{2}, j_{2}, c_{2}\right.$, curv $\left._{2}\right)$ (respectively), then there exists a unique natural equivalence $\psi: \hat{H}_{1} \rightarrow \hat{H}_{2}$ which is compatible with the given maps i.e. $\psi \circ i_{1}=$ $i_{2}, \psi \circ j_{1}=j_{2}, c_{2} \circ \psi=c_{1}$, and $\operatorname{curv}_{2} \circ \psi=\operatorname{curv}_{1}$.

Here, we observe that the following proposition is a direct consequence of Rakesh Pawar's results 112

Proposition 7. Let $M$ be a smooth manifold. Then there exists a group $G$ together with maps $i, j, c$, curv such that the following diagram commutes and has short exact diagonals :

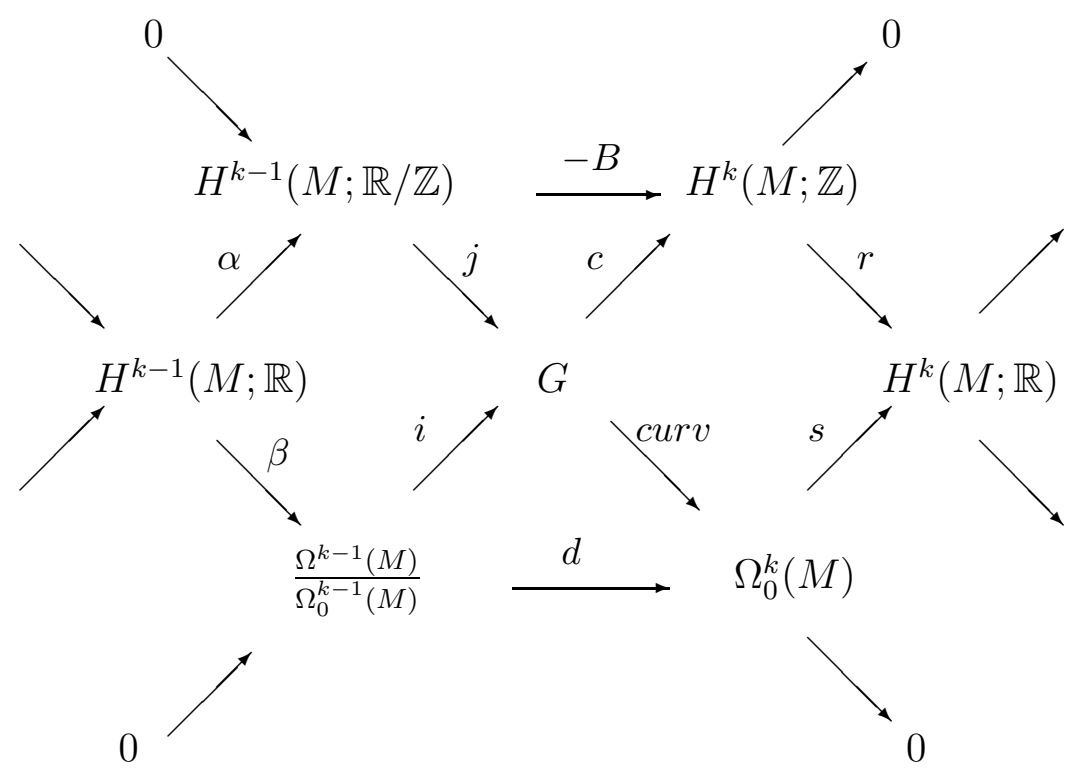

Furthermore, if $G^{\prime}$ is any other abelian group together with maps $i^{\prime}, j^{\prime}, c^{\prime}$, curv ${ }^{\prime}$ which make the diagram commute and have short exact diagonals, then there exists an isomorphism $\phi: G \rightarrow G^{\prime}$ such that $\phi \circ i=i^{\prime}, \phi \circ j=j^{\prime}, c^{\prime} \circ \phi=c$, and $c u r v v^{\prime} \circ \phi=c u r v$.

Proof. First, note that the diagram above can be redrawn [14] as : 


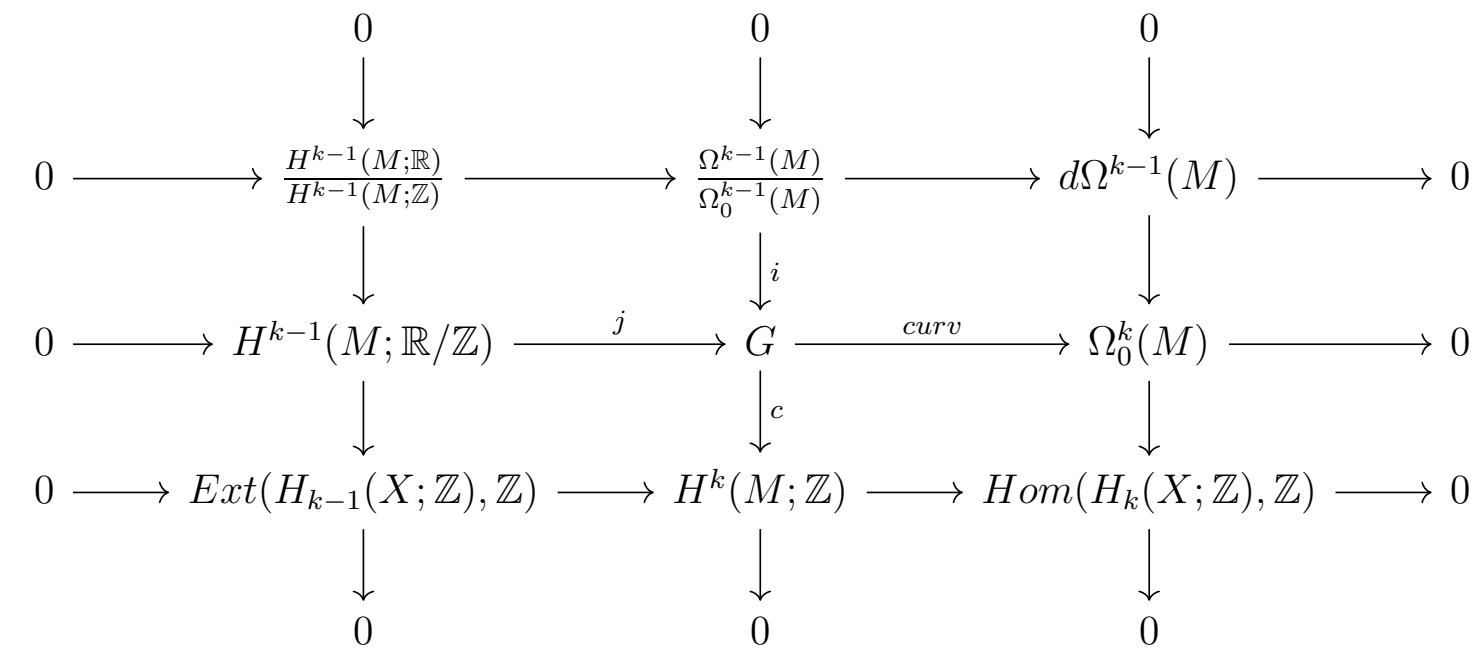

The proposition follows from corollary 4 by noting that $P=\frac{H^{k-1}(M ; \mathbb{R})}{H^{k-1}(M ; \mathbb{Z})}$ is divisible, and hence injective.

Note that the proposition 7 is weaker than the Simons-Sullivan theorem 6. The full Simons-Sullivan theorem is a statement about functors. We therefore consider the category of functors Fun $\left(\mathbf{M a n}^{o p}, \mathbf{A b}\right)$. This is an abelian category, having enough projectives and enough injectives [15, 16]. Therefore by proposition 3, we have the following

Proposition 8. The following are equivalent:

1. The Simons-Sullivan hexagon diagram uniquely determines the functor $\hat{H}^{n}(M ; \mathbb{R} / \mathbb{Z})$ upto a compatible natural equivalence.

2. The natural transformation $\operatorname{Hom}\left(d \Omega^{n-1}(-) \oplus \operatorname{Ext}\left(H_{n-1}(-; \mathbb{Z}), \mathbb{Z}\right), \frac{H^{n}(-; \mathbb{R})}{H^{n}(-; \mathbb{Z})_{\mathbb{R}}}\right) \stackrel{\alpha}{\rightarrow}$ $\operatorname{Ext}\left(\operatorname{Hom}\left(H_{n}(-, \mathbb{Z})\right), \frac{H^{n}(-; \mathbb{R})}{H^{n}(-; \mathbb{Z})_{\mathbb{R}}}\right)$ is an epimorphism in Fun $\left(\boldsymbol{M a n}^{o p}, \boldsymbol{A b}\right)$, and every natural transformation from $\operatorname{Hom}\left(H^{k-1}(-; \mathbb{R} / \mathbb{Z})+\frac{\Omega^{k-1}(-)}{\Omega_{0}^{k-1}(-)}\right)$ to $\frac{H^{n}(-; \mathbb{R})}{H^{n}(-; \mathbb{Z})_{\mathbb{R}}}$ which vanishes on $\frac{H^{n}(-; \mathbb{R})}{H^{n}(-; \mathbb{Z})_{\mathbb{R}}}$ extends to a natural transformation on $\hat{H}^{n}(-; \mathbb{R} / \mathbb{Z})$.

In this proposition, the hom sets are in the Fun $\left(\mathbf{M a n}^{o p}, \mathbf{A b}\right)$ category. The functor $\frac{H^{n}(-; \mathbb{R})}{H^{n}(-; \mathbb{Z})_{\mathbb{R}}}$ is considered as a subobject of the functor $H^{k-1}(-; \mathbb{R} / \mathbb{Z})+$ $\frac{\Omega^{k-1}(-)}{\Omega_{0}^{k-1}(-)}$ which in in turn a subobject of $\hat{H}^{n}(-; \mathbb{R} / \mathbb{Z})$.

As we have seen in the proof of proposition 7 the corresponding question in the category $\mathbf{A b}$ is trivial since $\frac{H^{n-1}(M ; \mathbb{R})}{H^{n-1}(M ; \mathbb{Z})_{\mathbb{R}}}$ is divisible and hence injective. However, it is difficult to see whether or not the functor $\frac{H^{n-1}(-; \mathbb{R})}{H^{n-1}(-; \mathbb{Z})_{\mathbb{R}}}$ is an injective object in Fun $\left(\mathbf{M a n}^{o p}, \mathbf{A b}\right)$. 


\subsection{The case of differential K-theory}

Complex topological K-theory too admits a differential refinement called differential K-theory. For a survey of various models of differential K-theory, see [17]. In [9], Simons and Sullivan develop a model of differential K-theory for compact manifolds as the Grothendieck completion of the semigroup of 'structured vector bundles' and show that this group fits into a hexagon diagram :

Proposition 9. The differential K-groups fit into the hexagon diagram

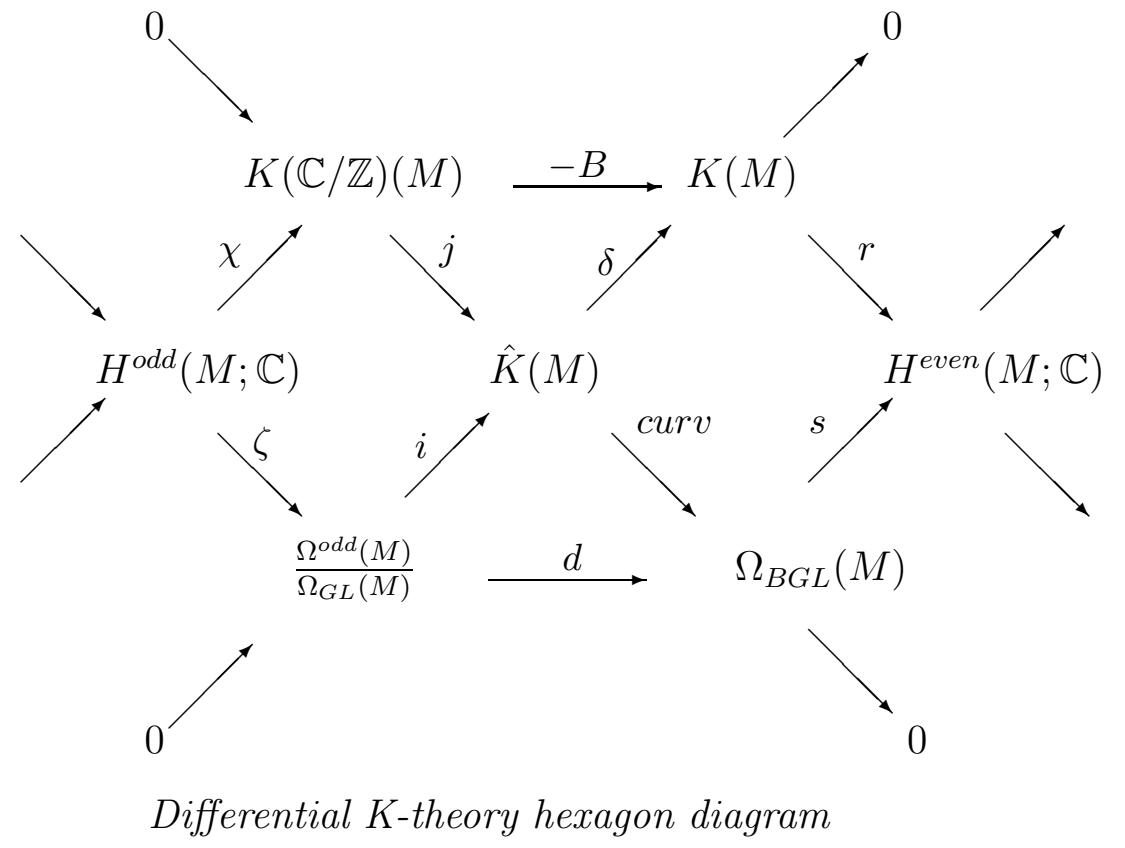

Here $\chi$ is reduction $\bmod \mathbb{Z}$, and $\zeta$ is induced by the de Rham map.

For a fuller description of the terms and maps in this diagram, see [9]. Throughout this subsection, i.e. in the context of differential $K$-theory, $M$ is assumed to be a compact manifold. Simons and Sullivan ask whether the diagram determines the groups $\hat{K}(M)$ upto isomorphism compatible with the other maps in the diagram. The following proposition provides a partial answer to their question.

Proposition 10. If $\hat{K}^{\prime}(M)$ is any other abelian group together with maps $i^{\prime}, j^{\prime}, \delta^{\prime}$, curv $^{\prime}$ which makes the above hexagon diagram commute, and have short exact diagonals, then there exists an isomorphism $\phi: \hat{K}(M) \rightarrow \hat{K}^{\prime}(M)$ such that $\phi \circ i=i^{\prime}, \phi \circ j=j^{\prime}, \delta^{\prime} \circ \phi=\delta$, and curv ${ }^{\prime} \circ \phi=c u r v$.

Proof. The diagram can be redrawn as 


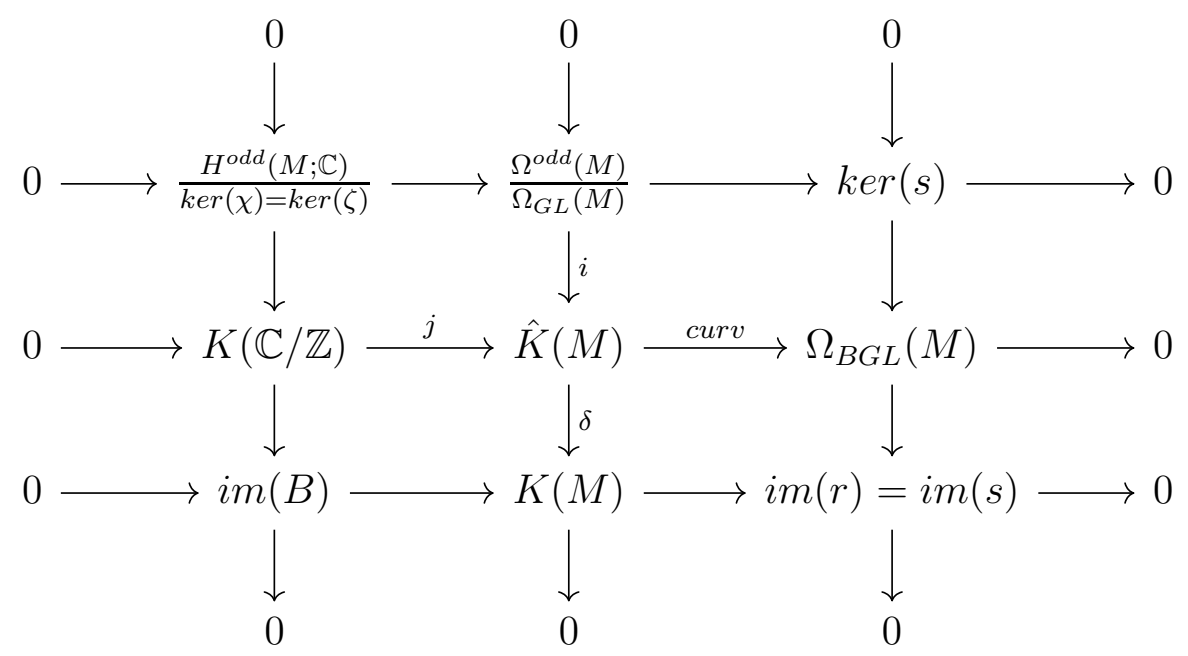

The proposition follows from corollary 4 by noting that $P=\frac{H^{\text {odd }}(M ; \mathbb{C})}{\operatorname{ker}(\chi)=\operatorname{ker}(\zeta)}$ is divisible and hence an injective object in $\mathbf{A b}$.

This is weaker than the claim of uniqueness of the functor $\hat{K}$. Consider the functor category Fun $\left(\mathbf{M a n}_{c p t}^{o p}, \mathbf{A b}\right)$. From 3, we note the following

Proposition 11. The following are equivalent :

1. If $\hat{K}^{\prime}(-)$ is another functor from $\boldsymbol{M a n}_{c p t}^{o p}$ to $\boldsymbol{A} \boldsymbol{b}$ together with natural transformations $i^{\prime}, j^{\prime}, \delta^{\prime}$, curv $v^{\prime}$ which fit in the hexagon diagram with exact diagonals, then there is a natural equivalence $\phi: \hat{K}(-) \rightarrow \hat{K}^{\prime}(-)$ such that $\phi \circ i=i^{\prime}, \phi \circ j=j^{\prime}, \delta^{\prime} \circ \phi=\delta$, and curv' $\circ \phi=$ curv.

2. The natural transformation $\operatorname{Hom}(R \oplus S, P) \stackrel{\alpha}{\rightarrow} \operatorname{Ext}^{1}(Q, P)$ is an epimorphism in Fun $\left(\boldsymbol{M a n}_{\text {cpt }}^{o p}, \boldsymbol{A b}\right)$, and every natural transformation from $E+H$ to $P$ which vanishes on $P$ extends to a natural transformation on $X$ where $P=\frac{H^{\text {odd }}(-; \mathbb{C})}{\operatorname{ker}(\chi)=\operatorname{ker}(\zeta)}, E=\frac{\Omega^{\text {odd }}(-)}{\Omega_{G L}(-)}, H=K(\mathbb{C} / \mathbb{Z})(-), R=$ $\operatorname{ker}(s), S=i m(B)$, and $X=\hat{K}(-) \in \operatorname{Fun}\left(\boldsymbol{M a n}_{c p t}^{o p}, \boldsymbol{A b}\right)$.

Like in proposition 8 , we consider $P$ as a subobject of $E+H$, which is in turn a subobject of $X$.

A stronger and more general result showing the uniqueness of the differential version of exotic cohomology theories with integration (and hence differential K-theory functor, in particular) has been proved in [11] by a different method.

\section{Acknowledgments}

I wish to thank my supervisor Dr. Rishikesh Vaidya for discussions and support. I am thankful to Jitendra Rathore for valuable discussions. I am 
grateful to the Council of Scientific \& Industrial Research for financial support under the CSIR-SRF(NET) scheme.

\section{References}

[1] J. Cheeger and J. Simons, "Differential characters and geometric invariants," in Geometry and Topology, pp. 50-80. Springer Berlin Heidelberg, Berlin, Heidelberg, 1985.

[2] P. Gajer, "Geometry of Deligne cohomology," Inventiones mathematicae 127 no. 1, (Jan, 1997) 155-207. https://doi.org/10.1007/s002220050118.

[3] J.-L. Brylinski, Loop Spaces, Characteristic Classes and Geometric Quantization, vol. 107 of Modern Birkhäuser Classics. Birkhäuser Basel, 1993.

[4] R. Harvey, B. Lawson, and J. Zweck, "The de Rham-Federer theory of differential characters and character duality," American Journal of Mathematics 125 no. 4, (2003) 791-847. http://doi.org/10.1353/ajm.2003.0025

[5] R. Harvey and B. Lawson, "From Sparks to Grundles — Differential Characters,"

Communications in Analysis and Geometry 14 no. 1, (2006) 25-58. https://dx.doi.org/10.4310/CAG.2006.v14.n1.a2.

[6] U. Bunke, M. Kreck, and T. Schick, "A geometric description of differential cohomology," Annales mathématiques Blaise Pascal 17 no. 1, (2010) 1-16. http://www. numdam.org/item/AMBP_2010__17_1_1_0.

[7] J. Simons and D. Sullivan, "Axiomatic characterization of ordinary differential cohomology," Journal of Topology 1 no. 1, (10, 2007) 45-56. https://doi.org/10.1112/jtopol/jtm006.

[8] M. Hopkins and I. Singer, "Quadratic functions in geometry, topology, and M-theory," J. Differential Geom. 70 no. 3, (07, 2005) 329-452, arXiv:math/0211216. https://doi.org/10.4310/jdg/1143642908.

[9] J. Simons and D. Sullivan, "Structured bundles define differential $K$-theory," in Géométrie différentielle, physique mathématique, 
mathématiques et société (I) : Volume en l'honneur de Jean Pierre Bourguignon, H. Oussama, ed., no. 321 in Astérisque, pp. 1-3.

SocSimilarily the iété mathématique de France, 2008.

http://www. numdam.org/item/AST_2008__321__1_0.

[10] R. Pawar, "A generalization of Grothendieck's extension Panachées," Proc Math Sci 129 (2019). https://doi.org/10.1007/s12044-019-0523-7.

[11] U. Bunke and T. Schick, "Uniqueness of smooth extensions of generalized cohomology theories,"

Journal of Topology 3 no. 1, (2010) 110-156. https://londmathsoc.onlinelibrary.wiley.com/doi/abs/10.1112/jtopol/jtq002.

[12] C. A. Weibel, An Introduction to Homological Algebra. Apr, 1994. /core/books/an-introduction-to-homological-algebra/AAA3F16482097015CD12D4376

[13] J. Rotman, An Introduction to Homological Algebra. Universitext. Springer-Verlag, New York, 2 ed., 2009. https://www . springer.com/gp/book/9780387245270.

[14] C. Bär and C. Becker, Differential Characters and Geometric Chains, pp. 1-90. Springer International Publishing, Cham, 2014. arXiv:1303.6457. "https://doi.org/10.1007/978-3-319-07034-6_1".

[15] K. A. (https://math.stackexchange.com/users/31228/kevin arlin), "About functor category and enough injectives." Mathematics stack exchange. https://math.stackexchange.com/q/2460009. URL:https://math.stackexchange.com/q/2460009 (version: 2017-10-06).

[16] Z. L. (https://mathoverflow.net/users/11640/zhen lin), "Projectives and Injectives in Functor Categories." Mathoverflow. https://mathoverflow.net/q/162801. URL:https://mathoverflow.net/q/162801 (version: 2016-01-23).

[17] U. Bunke and T. Schick, "Differential K-Theory: A Survey," in Global Differential Geometry, C. Bär, J. Lohkamp, and M. Schwarz, eds., pp. 303-357. Springer Berlin Heidelberg, Berlin, Heidelberg, 2012.

Ishan Mata, Department of Physics, Birla Institute of Technology and Science - Pilani, Pilani campus, Pilani, Rajasthan, INDIA. PIN : 333031

Email : ishanmata@gmail.com 